\title{
Fast $r$-adaptivity for multiple queries of heterogeneous stochastic material fields
}

\author{
R. Cottereau · P. Díez
}

Received: date / Accepted: date

\begin{abstract}
We present an $r$-adaptivity approach for boundary value problems with randomly fluctuating material parameters solved through the Monte Carlo or stochastic collocation methods. This approach tailors a specific mesh for each sample of the problem. It only requires the computation of the solution of a single deterministic problem with the same geometry and the average parameter, whose numerical cost becomes marginal for large number of samples. Starting from the mesh used to solve that deterministic problem, the nodes are moved depending on the particular sample of mechanical parameter field. The reduction in the error is small for each sample but sums up to reduce the overall bias on the statistics estimated through the Monte Carlo scheme. Several numerical examples in 2D are presented.
\end{abstract}

Keywords Stochastic mechanics - Monte Carlo method S Stochastic collocation method $\cdot$ Error Estimation $\cdot r$-adaptivity

\section{Introduction}

In a large number of scientific domains, random fields are routinely used to quantify uncertainties and parameterize fluctuating properties. These domains include micromechanics $(32 ; 24 ; 43 ; 36 ; 20)$, geomechanics and seismic engineering (47; $21 ; 54 ; 45 ; 2)$, modeling of concrete and quasi-brittle materials $(56 ; 33)$, geophysics $(27 ; 30 ; 53 ; 50 ; 9)$, structural mechanics $(17 ; 19)$, computational fluid dynamics $(18 ; 37)$, dynamics of chemical reactions (23), and many others. Various

\section{R. Cottereau}

Laboratoire MSSMat UMR 8579, École Centrale Paris, CNRS,

Châtenay-Malabry, France

E-mail: regis.cottereau@ecp.fr

P. Díez

Laboratori de Càlcul Numèric,

Departament de Matemàtica Aplicada III, Universitat Politècnica de Catalunya,

International Center for Numerical Methods in Engineering (CIMNE),

C/Jordi Girona 1-3, Campus Nord, Edifici C2, E-08034 Barcelona, Spain 
Galerkin methods, such as the stochastic finite element method $(28 ; 7 ; 52 ; 37)$, have been developed to approximate numerically the solutions of these problems. These methods are rather straightforward extensions of their deterministic counterparts, due to the tensor structure of the functional spaces considered, but the resulting systems to be inverted are extremely large. In the continuity of error estimation for deterministic problems $(5 ; 6 ; 58 ; 1 ; 35 ; 55 ; 8)$, the numerical analysis for these stochastic problems has been widely discussed, either through $a$ priori $(12 ; 4 ; 26 ; 10 ; 14 ; 51 ; 57 ; 15)$, a posteriori $(22 ; 40 ; 13)$, or goal-oriented estimates $(42 ; 34 ; 41 ; 11 ; 25)$.

The Monte Carlo method $(12 ; 49)$ provides an alternative to these spectral approaches. It consists in generating samples of the random material parameter field and approximating the statistics of a quantity of interest as a combination of those obtained for each sample independently. Numerically, this method therefore reduces to the approximation of a series of deterministic partial differential equations. Although there might be a very large number of these problems, the Monte Carlo method remains a method of choice for at least two reasons: (i) the rate of convergence of the solutions is independent of the dimensionality of the stochastic space, and (ii) it allows to inherit whatever numerical tools were constructed over the years for the particular physical problem at hand. The last remark extends to collocation, sparse grid and other similar methods (37, Chapter 3), as they also result in multiple queries of the similar numerical solver. Specific error estimators have been designed for the Monte-Carlo method $(12 ; 25 ; 15)$ and the collocation method $(4 ; 26 ; 14 ; 57)$. We concentrate in this paper on improving simulations performed with the Monte Carlo, collocation, sparse grid or similar techniques.

In practice, the different instances of the Monte Carlo samples are typically approximated through the Finite Element (FE) method or another similar variational method. In that case, the same mesh is normally used for all realizations of the parameter field and that mesh is designed to provide a reasonable accuracy in the case of the average material field. It therefore does not account for the actual realization of the stochastic material field. The possibility of designing a new mesh from scratch for each Monte Carlo realization is not reasonable. Indeed, meshing potentially involves a lot of human interaction and is always computationally intensive for industrial problems.

An alternative is the $r$-adaptivity technique $(31 ; 3 ; 38 ; 16 ; 46 ; 48 ; 39 ; 29)$. It relies on the deformation of an initial mesh, based on appropriate error indicators. As the connectivity is not modified, most of the meshing issues are hidden in the construction of that one initial mesh. However, it is limited to small modifications of the initial mesh. Otherwise, some element shapes might make the mesh unfit for FE approximations. Note that this is exactly the kind of problems we are considering here, where the random parameter fields are fluctuations around a known average field and at least part of the complexity of the solution (and hence of the complexity of the mesh required for a good approximation) comes from the geometry and the loading, rather than from the parameter field.

The objective of this paper is therefore to develop such an $r$-adaptivity strategy for Monte Carlo (and similar) solutions of boundary value problems with random parameters. Two ingredients have to be developed: (i) an error estimator depending on each realization of the parameters (this is described in Section 3.1) and (ii) a method to move the nodes of the initial mesh for a given error map (this is described in Section 3.3). For the method to remain numerically affordable, 
the error estimate (i) must be explicit because it has to be computed for each realization. Likewise, the numerical cost of the adaptation method (ii) must be limited. Besides the core Section 3, Section 2 presents the problem to be solved and Section 4 introduces a series of $2 \mathrm{D}$ numerical examples. The first example (Section 4.1) discusses the efficiency of the mesh adaptation, as well as the values of a scaling parameter that it involves. The following two examples show the efficiency of the method.

\section{Problem statement}

In this section, we introduce the boundary value problem of interest, with a stochastic heterogeneous material parameter, as well as the corresponding (deterministic) average problem, where the material field is replaced by its average. We also introduce some notations related to the numerical approximations and solutions of these two problems.

2.1 Boundary value problem with random heterogeneous parameter

We consider the Poisson equation, whose strong form reads: find $u$ taking values in an open domain $\Omega$ such that:

$$
\begin{aligned}
-\nabla \cdot(a \nabla u) & =f & & \text { in } \Omega \\
(a \nabla u) \cdot \boldsymbol{n} & =g_{N} & & \text { on } \Gamma_{N} \\
u & =u_{D} & & \text { on } \Gamma_{D}
\end{aligned}
$$

The Dirichlet boundary $\Gamma_{D}$ and Neumann boundary $\Gamma_{N}$ form a partition of the boundary of the domain $\partial \Omega$.

The material parameter is assumed to be a random field in space. We therefore introduce the complete probability space $(\Theta, \mathcal{T}, P)$, where $\Theta$ is a set of outcomes, $\mathcal{T}$ is a $\sigma$-algebra of events in $\Theta$ and $P: \mathcal{T} \rightarrow[0,1]$ is a probability measure. With that definition, the parameter field $a(\boldsymbol{x}, \boldsymbol{\theta})$ is variable in space and also depends on the random parameter $\boldsymbol{\theta} \in \Theta$. All equalities in the strong formula should be considered as true $P$-almost surely. We denote $a(\boldsymbol{x}, \boldsymbol{\theta})=a_{0}+\delta a(\boldsymbol{x}, \boldsymbol{\theta})$, where the average $a_{0}$ is deterministic and the average of the fluctuation $\delta a(\boldsymbol{x}, \boldsymbol{\theta})$ vanishes at every $\boldsymbol{x} \in \Omega$. Note that the contents of this paper also apply when the average parameter $a_{0}$ is fluctuating in space, and we consider it here constant for simplicity only.

\subsection{Average boundary value problem}

We now consider the average boundary value problem, which is similar to the previous one, except that the material parameter is $a_{0}$. As this field is deterministic, so is the boundary value problem and its solution. The strong form reads: find $u_{0}$ 
taking values in $\Omega$ such that:

$$
\begin{aligned}
-\nabla \cdot\left(a_{0} \nabla u_{0}\right) & =f & & \text { in } \Omega \\
\left(a_{0} \nabla u_{0}\right) \cdot \boldsymbol{n} & =g_{N} & & \text { on } \Gamma_{N} \\
u_{0} & =u_{D} & & \text { on } \Gamma_{D}
\end{aligned}
$$

Finally, we decompose the solution $u(\boldsymbol{x}, \boldsymbol{\theta})$ of (1) as the solution of the average problem (2) and a perturbation: $u(\boldsymbol{x}, \boldsymbol{\theta})=u_{0}(\boldsymbol{x})+\delta u(\boldsymbol{x}, \boldsymbol{\theta})$.

\subsection{Numerical approximation}

The solution of the average problem (2) is approximated with a FE scheme using a mesh with characteristic size $H$. The approximation of the solution is denoted $u_{0}^{H}$.

The solution of the stochastic problem (1) is approximated using the Monte Carlo method (or a similar method, as explained in the introduction). A series of $N$ samples $\left\{a\left(\boldsymbol{x}, \boldsymbol{\theta}_{i}\right)\right\}_{1 \leq i \leq N}$ of the parameter field $a(\boldsymbol{x}, \boldsymbol{\theta})$ are then chosen. Typically the same mesh as before (with characteristic size $H$ ) is used to solve the (deterministic) boundary value problems for each of the samples. A series of $N$ solutions $\left\{u^{H}\left(\boldsymbol{x}, \boldsymbol{\theta}_{i}\right)\right\}_{1 \leq i \leq N}$ are then obtained as an approximation $u^{H}(\boldsymbol{x}, \boldsymbol{\theta})$ of the solution $u(\boldsymbol{x}, \boldsymbol{\theta})$.

Each of the $N$ FE solutions comes with a certain numerical error. Classical techniques exist to minimize this error by adapting the mesh, adding or merging elements where needed. This may however become costly when implemented within a Monte Carlo loop. We therefore restrict ourselves to an $r$-adaptive scheme only, which only moves the nodes of the mesh, without changing the connectivity. The goal is to eventually reduce at a marginal cost the error for each of the approximate solutions $\left\{u^{H}\left(\boldsymbol{x}, \boldsymbol{\theta}_{i}\right)\right\}_{1 \leq i \leq N}$ and therefore also for the approximate solution $u^{H}(\boldsymbol{x}, \boldsymbol{\theta})$.

\section{An $r$-adaptive strategy based on an error indicator for the perturbed problem}

As stated above, we aim at adapting the mesh in each of the $N$ samples. The $r$-adaptation leaves low flexibility in modifying the mesh because the connectivity is unchanged and the distortion must be limited to avoid pathological meshes. Moreover, the motion of the boundary nodes is constrained to keep the nodes on the boundary.

An adaptive strategy is readily identified by describing (i) the error assessment strategy and (ii) the remeshing criterion. That is, (i) how to assess the error in each zone of the domain in order to increase the numerical resolution where the error is larger and (ii) how to modify (adapt) the mesh, based on the information provided by the error assessment.

This has to be complemented with an algorithm that constructs the new (adapted) mesh, satisfying the conditions enforced by the remeshing criterion. That is, respecting the desired element sizes. In the particular case of $r$-adaptivity, the algorithm to move the mesh consists in expanding some elements (where the 
error is expected to be smaller) and contracting the others (where the error is expected to be larger). Thus, the resulting $r$-adapted mesh is going to concentrate nodes and enrich the discretization in the zones where the solution is difficult to resolve.

Note that the accuracy improvement associated with this adaptive strategy is not expected to be very important for each sample. Therefore, the computational cost of the proposed approach must be also marginal, in comparison with that of a standard FE solver. This suggests using an a priori error indicator, based on the variation of the input data $\delta a$ for each sample.

\subsection{Error indicator approximating the Laplacian}

The standard a priori estimates for linear FE formulations state that the associated errors (measured in some norm) are bounded by some power of $H$, affected by a constant proportional to the second derivatives of the solution. The second derivatives are contained in the Hessian matrix and fairly well represented by its trace, the Laplacian $\Delta u$.

Following this rationale, geometrical interpolation error indicators are based on the estimated values of the second derivatives of the solution. This is a classical approach (see for instance (44)) in an a posteriori setup, that is estimating the second derivatives of the solution $u$ after having computed a previous approximation $u^{H}$ that has to be improved. The error indicator is in this case recovered as a post-process of $u^{H}$.

Here we aim at computing an approximation to $\Delta u$ using the information at hand, that is $u_{0}, a_{0}$ and $\delta a$, without computing any approximation $u^{H}$ to the solution of the perturbed problem, $u=u_{0}+\delta u$.

The proposed, a priori computable approximation of the Laplacian, $\xi$, reads

$$
\Delta u \approx \xi:=-\frac{1}{a_{0}}\left[f\left(1-\frac{\delta a}{a_{0}}\right)+\nabla \delta a \cdot \nabla u_{0}\right]
$$

The expression for $\xi$ is derived considering that (1a) is equivalent to

$$
-\nabla \cdot\left(\left(a_{0}+\delta a\right) \nabla\left(u_{0}+\delta u\right)\right)=f
$$

Thus, using (2a) (multiplying both sides by $\delta a: \delta a \Delta u_{0}=-\frac{\delta a}{a_{0}} f$ ) and considering that the product of two perturbed quantities (quadratic) is negligible in front of linear perturbations, (3) is readily recovered by considering that

$$
\begin{aligned}
-a_{0} \Delta u=-a_{0} \nabla \cdot \nabla\left(u_{0}+\delta u\right) & =f+\nabla \cdot\left(\delta a \nabla\left(u_{0}+\delta u\right)\right. \\
& =f+\nabla \cdot\left(\delta a \nabla u_{0}\right)+\underbrace{\nabla \cdot(\delta a \nabla \delta u)}_{\approx 0} \\
& \approx f+\nabla \delta a \cdot \nabla u_{0}+\underbrace{\delta a \nabla \cdot \nabla u_{0}}_{=-\frac{\delta a}{a_{0}} f} \\
& =f\left(1-\frac{\delta a}{a_{0}}\right)+\nabla \delta a \cdot \nabla u_{0}
\end{aligned}
$$

Note that the expression for $\xi$ in (3) is fully computable once the perturbation $\delta a$ associated with each sample is provided. This quantity has to be computed 
element wise, $\xi_{e}$ for $e=1, \ldots, n_{\mathrm{el}}$, being $n_{\mathrm{el}}$ the number of elements in the mesh. The cost of computing $\xi_{e}$ is low because the only two terms that vary along the sampling process are $\delta a$ and $\nabla \delta a$, that have to be evaluated at some representative point of each element.

\subsection{Remeshing criterion}

Recall that, in the current framework, adaptivity consists in building a tailored mesh for sample $i, i=1, \ldots, N$, as a modification of the mesh designed for the non perturbed average problem. Thus, the goal of the remeshing criterion is to determine the desired element size $h_{e}$ in the zone occupied in the average mesh by element $\Omega_{e}$ of size $H_{e}$, for $e=1, \ldots, n_{\mathrm{el}}$.

The curvature (Laplacian) of $u$ in element $\Omega_{e}$ is estimated by a mean value $\xi_{e}$, which is computed using (3) and evaluating $f, \delta a$ and $\nabla u_{0}$ at some representative points of element $\Omega_{e}$.

The mesh is designed such that the element size in the zone of $\Omega_{e}$ is inversely proportional to the curvature, that is

$$
h_{e}=\frac{1}{\frac{1}{h_{\max }}+\xi_{e} \alpha}
$$

where the parameter $h_{\max }$ is an upper limit for $h_{e}$ and $\alpha$ is a normalization parameter. Typically, $h_{\max }$ is selected lower than twice the characteristic element size of the initial mesh, $H$, in order to avoid distortions in the resulting mesh. Then, $\alpha$ is characterized to obtain the desired number of elements in the resulting mesh. In the case of $r$-adaptivity, the number of elements in the initial and the resulting meshes are equal (to $n_{\mathrm{el}}$ ) and therefore $\alpha$ is such that

$$
\sum_{e=1}^{n_{\mathrm{el}}} \frac{h_{e}^{2}}{2}=\sum_{e=1}^{n_{\mathrm{el}}}\left|\Omega_{k}\right| .
$$

This expression stands for triangular elements, assuming that the area of the triangle is $\frac{h_{e}^{2}}{2}$.

The required deformation (extension or contraction) of element $\Omega_{e}$ is therefore defined by the ratio

$$
\varepsilon_{e}=\frac{h_{e}}{H_{e}}, \text { for } e=1, \ldots, n_{\mathrm{el}}
$$

\subsection{Moving the mesh}

The prescribed element size is made relative to the original mesh designed for the average value of $a, a_{0}$. Thus, the original mesh has to be deformed in order to accommodate the desired element sizes $h_{e}$, for $e=1, \ldots, n_{\mathrm{el}}$. The deformation factors $\varepsilon_{e}$ for each element $\Omega_{e}$ of the mesh given in (6) are computable once the error indicator $\xi_{e}$ is obtained using (3).

In the $r$-adaptive framework, mesh deformation consists in relocating the nodes and modifying the element without altering the connectivity. As previously noted, 
this does not allow drastic changes in the configuration of the mesh. Nevertheless, as will be shown in the examples, the modest accuracy improvement in each simulation along the Monte Carlo or Collocation sampling results in an overall improvement of the stochastic characterization. In this context, the proposed technique to relocate the nodes has to be simple and computationally efficient. The motion of the nodes is characterized by a vector of nodal displacements $\delta \boldsymbol{x}$. The marginal accuracy gain expected in the adaptive process suggests selecting a low-cost algorithm to determine $\delta \boldsymbol{x}$.

The technique adopted here consists in solving a linear elastic mechanical problem to obtain the displacement of the nodes $\delta \boldsymbol{x}$. The loading is given by an isotropic prescribed strain in each element, precisely $\varepsilon_{e}, e=1, \ldots, n_{\mathrm{el}}$. To further simplify the formulation, the mechanical model is selected with Lamé coefficients $\mu=1 / 2$ and $\lambda=0$ (corresponding to $E=1$ and $\nu=0$ ). Thus, the pre-stress is, at each element $\varepsilon_{e} \mathbf{I}$. The equation to be solved for the nodal displacements $\delta \boldsymbol{x}$ taking values in $\Omega$ is then

$$
\nabla \cdot \nabla_{\mathrm{s}} \delta \boldsymbol{x}=\nabla \cdot(\varepsilon \mathbf{I})=\nabla \varepsilon
$$

to be complemented with the proper boundary conditions enforcing the geometrical restrictions, that is guaranteeing that boundary nodes do not leave the boundary.

Note that the computational effort to obtain $\delta \boldsymbol{x}$ at each of the $N$ samples is very low because it is computed with the initial mesh and therefore the stiffness matrix associated with problem (7) is the same for all the samples. Thus, the matrix is factorized once and the cost of obtaining $\delta \boldsymbol{x}$ is just the cost of computing the force term associated with the right-hand-side, $\nabla \varepsilon$, plus a matrix-vector product corresponding to the forward and backward substitutions. Note also that the cost of computing the force term is low because it sums up to obtaining nodal values for $\nabla \varepsilon$ (from the elementary values $\varepsilon_{e}$ ) and premultiplying them by a mass matrix (again, the same for all the samples).

\subsection{Extension to second-order tensor-valued coefficients}

Until now, we have only considered scalar coefficients $a(\boldsymbol{x}), a_{0}$ and $\delta a(\boldsymbol{x})$. For many applications of interest, this is not sufficient, and tensor-valued coefficients should be considered. We discuss in this section this extension of the results presented above to that case. Being a second order tensor, we now denote the coefficients with bold face fonts: $\boldsymbol{a}(\boldsymbol{x}), \boldsymbol{a}_{0}$ and $\delta \boldsymbol{a}(\boldsymbol{x})$. We further assume that the tensors $\boldsymbol{a}$ and $\boldsymbol{a}_{0}$ are positive definite, which is appropriate for many applications, and decompose the latter along its principal directions: $\boldsymbol{a}_{0}=\sum_{i} \lambda_{i} \boldsymbol{v}_{i} \otimes \boldsymbol{v}_{i}$, being $\boldsymbol{v}_{i}, i=1, \ldots, d$, the unit vectors describing the principal directions and $d$ the dimension of space considered. Equation (2a) now reads $-\boldsymbol{a}_{0}:(\nabla \otimes \nabla) u_{0}=-\nabla \cdot\left(\nabla \cdot\left(\boldsymbol{a}_{0} u_{0}\right)\right)=f$. In this case, the role played by the Laplacian in the isotropic case (average of second order derivatives in the principal directions) is played by the quantity

$$
\frac{1}{\operatorname{tr} \boldsymbol{a}_{0}} \boldsymbol{a}_{0}:(\nabla \otimes \nabla) u=\frac{1}{\sum_{i=1}^{d} \lambda_{i}} \sum_{i=1}^{d} \lambda_{i} \frac{\partial^{2} u}{\partial v_{i}^{2}}
$$

Note that for isotropic remeshing, the indicator is not expected to provide information on the critical directions because the adapted mesh is not going to 
be stretched along these directions. Therefore, this weighted average is providing meaningful information to decide which are the zones where the elements have to be isotropically expanded or contracted.

Thus, the indicator $\xi$ approximating this quantity (replacing Equation (3)) reads

$$
\xi:=-\frac{d}{\operatorname{tr} \boldsymbol{a}_{0}}\left[f\left(1-\frac{1}{d} \sum_{i=1}^{d} \frac{1}{\lambda_{i}} \boldsymbol{v}_{i} \cdot \delta \boldsymbol{a} \cdot \boldsymbol{v}_{i}\right)+(\nabla \cdot \delta \boldsymbol{a}) \cdot \nabla u_{0}\right] .
$$

Equation (8) is derived by considering that

$$
-\nabla \cdot \boldsymbol{a}_{0} \nabla u=f+\delta \boldsymbol{a}:(\nabla \otimes \nabla) u_{0}+(\nabla \cdot \delta \boldsymbol{a}) \cdot \nabla u_{0},
$$

and is equivalent to Equation (3) when $\boldsymbol{a}_{0}=a_{0} \mathbb{I}\left(\lambda_{i}=1, i=1, \ldots, d\right)$ and $\delta \boldsymbol{a}=\delta a \mathbb{I}$, where $\mathbb{I}$ is the identity tensor.

\section{Examples}

In this section, we present three examples that illustrate the behavior of our error indicator and of the $r$-adaptivity process. We first present two simple deterministic examples on which we analyse precisely the behavior of the adaptivity scheme proposed for single realizations of material property maps. The last example considers stochastic properties and illustrates the improvement introduced by the method for the evaluation of statistical quantities of interest.

\subsection{D fluctuation of properties}

We first consider a square domain $\Omega=[0,1] \times[0,1]$ and a material property map in the form $a(x)=a_{0}+\cos (2 \pi N x)$, where $a_{0}=2$ and $N$ is a positive integer. The corresponding maps of property are plotted in Figure 1. Homogeneous Dirichlet boundary conditions are considered on the left and right sides $(x=0$ and $x=1$ ) and homogeneous Neumann boundary conditions are considered elsewhere. A volume load is considered, with the form $f(x)=x^{2}-1 / 3$. Thanks to the form of the boundary conditions, force and material property, the solution of Problem (1) is quasi-1D and independent of the variable $y$. We compute for this problem a reference solution on a refined mesh with 360,000 elements, and denote it $u_{\text {ref }}(x)$.

The objective of this example is to consider two reasonable indicators of the error and combine them with two different strategies for moving the mesh. The two indicators are respectively: (i) based on the curvature of the exact solution $u_{\text {ref }}^{\prime \prime}=$ $\partial^{2} u_{\text {ref }} / \partial x^{2}$ and (ii) the indicator developed in this paper. The choice of $u_{\text {ref }}^{\prime \prime}=$ $\partial^{2} u_{\text {ref }} / \partial x^{2}$ as an indicator is based on the fact that, for linear finite elements, only one element is required to well approximate a linear solution. The two strategies are: (i) moving the $x$-coordinates of the nodes so that the distance along $x$ between two consecutive nodes is equal to the value computed in Eq. (5) and (ii) move the nodes in the mesh according to the strategy presented in Section 3.3. These two adaptivity strategies are essentially $1 \mathrm{D}$ and $2 \mathrm{D}$, respectively, and will be denoted as such.

We therefore consider in the following computations three different strategies: 

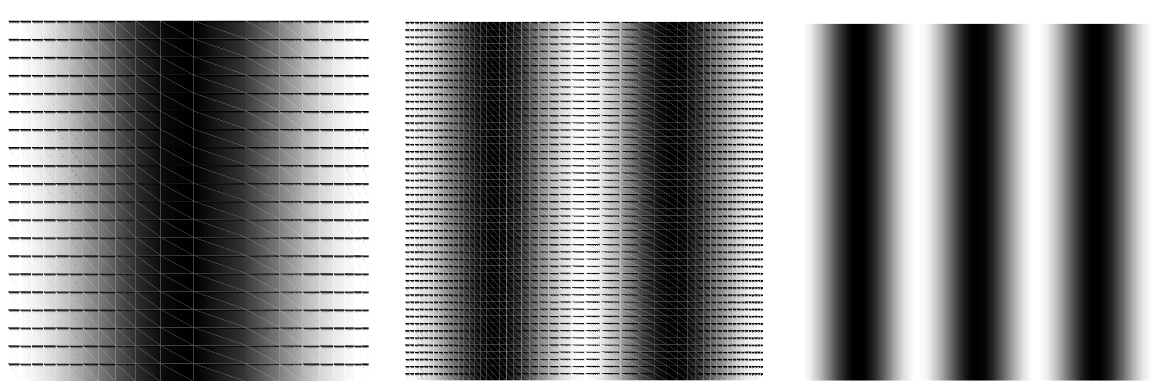

Fig. 1 Map of parameter $a(x)=a_{0}+\cos (2 \pi N x)$ for the three cases considered: (left) $N=1$, (center) $N=2$ and (right) $N=3$. White corresponds to $a(x)=3$ and black corresponds to $a(x)=1$.

1. use the indicator based on $u_{\text {ref }}^{\prime \prime}$ and the $1 \mathrm{D}$ adaptivity process;

2. use the indicator based on $u_{\text {ref }}^{\prime \prime}$ and the $2 \mathrm{D}$ adaptivity process;

3. use the indicator proposed in this paper and the $2 \mathrm{D}$ adaptivity process;

Note that the reference solution $u_{\text {ref }}(x)$ is not available in real applications so only the last one of the three proposed technique can then be applied. The introduction of the first two strategies is performed only for comparison purposes. For illustration, we plot in Figure 2 maps of element areas obtained with $N=2$, 2,500 elements, $h_{\max }=1.2$, and the three adaptive strategies. It is clear that the three strategies yield similar deformed mesh, although the mesh obtained with the 1D adaptivity process is more sharply defined than the other two. For those, the features are more smeared and transitions between small and large elements are smoother.
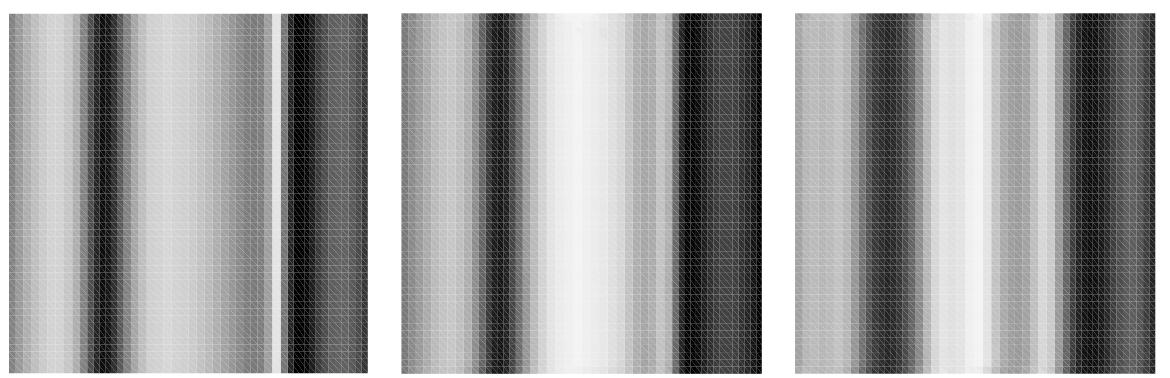

Fig. 2 Map of element areas obtained with $N=2,2,500$ elements, $h_{\max }=1.2$, and the three adaptive strategies: (left) the reference solution $u_{\text {ref }}^{\prime \prime}$ and the 1D adaptive strategy, (center) the reference solution $u_{\text {ref }}^{\prime \prime}$ and the $2 \mathrm{D}$ adaptive strategy, and (right) the proposed error indicator and the $2 \mathrm{D}$ adaptive strategy. White corresponds to $S=1.5 \times 10^{-4}$ and black corresponds to $S=2.5 \times 10^{-4}$

We now turn to the question of choosing the value of $h_{\max }$. We consider three different cases: (i) $N=1$, where $\Omega$ is discretized with 400 elements, (ii) $N=2$, where $\Omega$ is discretized with 2,500 elements, and (iii) $N=3$, where the domain is discretized with 10,000 elements. For each of these three meshes, we compute the 
errors in energy norm (with respect to the reference solution $u_{\text {ref }}$ ) obtained using the three different strategies described above for different values of the chosen maximum element size $h_{\max }$. The results are plotted in Figure 3 . In these figures, the errors are normalized with respect to the error obtained for a homogeneous mesh (that is to say, without moving the nodes). The maximum element sizes plotted in abscissa are equal to $\max h=\sqrt{2 \max S}$, where $S$ is the area of an element, and normalized with respect to the size of an element of the homogeneous mesh. Note that the normalized maximum element size is not $h_{\max }$, but the one that is obtained as output of the adaptivity process.
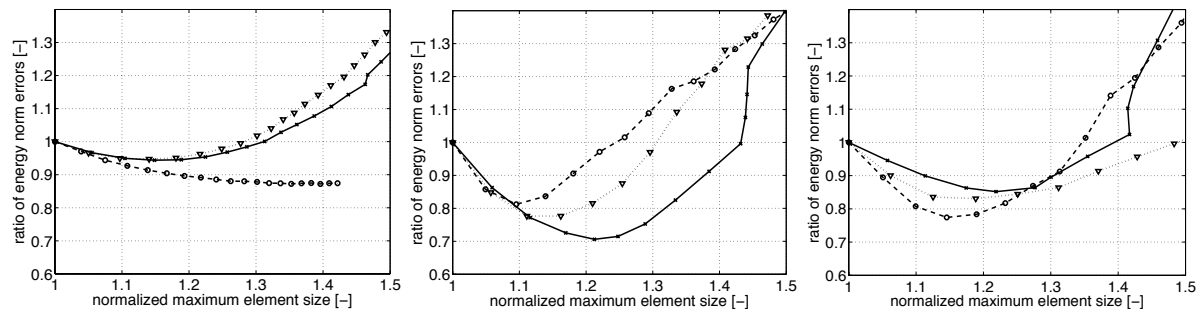

Fig. 3 Comparison of the evolution of the normalized error with respect to the normalized maximum element size using: (i) the reference solution $u_{\text {ref }}^{\prime \prime}$ and the 1D adaptive strategy (dashed lines and circles), (ii) the reference solution $u_{\mathrm{ref}}^{\prime \prime}$ and the 2D adaptive strategy (dotted lines and triangles), and (iii) the proposed error indicator and the 2D adaptive strategy (solid lines and crosses). The three figures correspond to the three cases: (left) $N=1$, (center) $N=2$ and (right) $N=3$.

The following comments can be made on the results plotted in Figure 3:

- All three refinement strategies seem to perform better than the homogeneous case (the relative error is lower than 1) for small values of the maximum element size;

- For large values of the maximum element size, the approximations obtained with the adapted mesh are worst than those obtained with the homogeneous mesh;

- There is no clear pattern as to which method of the three performs better, depending on the fluctuations of the properties with respect to those of the force.

As the first two strategies are based on the knowledge of the exact solution, we could expect that they should yield better approximations than the third. However, the degradation of the expected behavior is due to the adaptivity process. When the mesh moves, the evaluation of the local indicators should be updated lest this movement be too extreme.

The conclusion of this first example is that, on such a simple configuration at least, our proposed strategy (combining the error indicator and the adaptivity process) seems to work well for a large range of desired maximum element size and situations. 


\section{$4.22 \mathrm{D}$ fluctuation of properties}

We consider here the same square domain $\Omega=[0,1] \times[0,1]$ as before, and a material property map in the form $a(x)=a_{0}+\cos (6 \pi x) \cos (6 \pi y)$, where $a_{0}=2$ (see Figure 4). As before, homogeneous Dirichlet boundary conditions are considered on the left and right sides $(x=0$ and $x=1)$ and homogeneous Neumann boundary conditions are considered elsewhere. The same volume load is considered $f(x)=x^{2}-1 / 3$. This time, because of the form of the fluctuations of the material properties field, the solution if fully 2D. As before, we compute a reference solution on a refined mesh with 360,000 elements, and denote it $u_{\text {ref }}(x)$. We also compute a reference solution considering the exact same problem, but a homogeneous field of material parameter at the value $a_{0}$. This solution is denoted $u_{\mathrm{ref}_{0}}$.

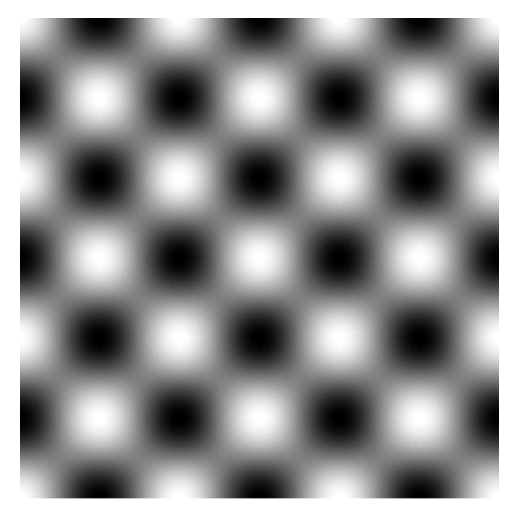

Fig. 4 Map of parameter $a(x)=a_{0}+\cos (6 \pi x) \cos (6 \pi y)$. White corresponds to $a(x)=3$ and black corresponds to $a(x)=1$.

The objective of this example is to observe the behavior of the proposed adaptivity process, and check its convergence rate with respect to the number of degrees of freedom (DOFs). We will compute this convergence rate for four different types of meshes: (i) a homogeneous mesh (for which theoretical convergence rates are known), (ii) a mesh deformed with respect to the curvature $u_{\text {ref }_{0}}^{\prime \prime}$ of the reference solution for a homogeneous material parameter, (iii) a mesh deformed with respect to the curvature $u_{\text {ref }}^{\prime \prime}$ of the reference solution, and (iv) a mesh deformed with the proposed adaptivity process. Note that the mesh (ii) is expected to propose refinement based only on the information of the loading or boundary conditions, because it does not bear any information on the field $a(x)$. As the solution is fully $2 \mathrm{D}$, all these meshes have been obtained using the $2 \mathrm{D}$ adaptivity process described in Section 3.3. For illustration, we plot in Figure 5 maps of element areas obtained with 2,500 elements, $h_{\max }=1.5$, and the three adaptive strategies (ii), (iii) and (iv). It is clear that the last two yield similar deformed mesh. The first one shows a $1 \mathrm{D}$ deformation because boundary conditions and loading are essentially 1D. Note that both the meshes (iii) and (iv) are extremely complex and could not have been simply guessed.

We now consider the convergence rate of the error computed using these four meshing strategies with respect to the number of DOFs. These errors are plotted 

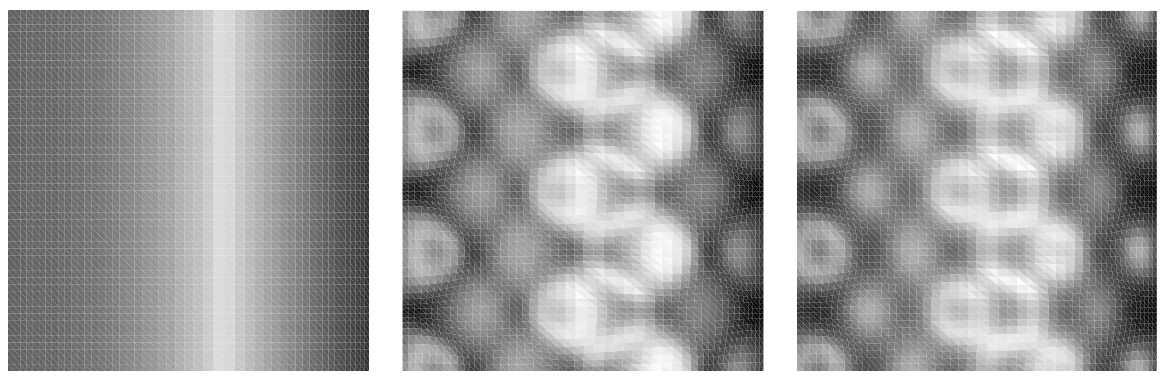

Fig. 5 Map of element areas obtained with 2,500 elements, $h_{\max }=1.5$, and the three adaptive strategies, using: (left) the solution $u_{\text {ref }_{0}}^{\prime \prime}$ for average property field $a_{0}$, (center) the reference solution $u_{\text {ref }}^{\prime \prime}$ and (right) the proposed error indicator. White corresponds to $S=3.5 \times 10^{-4}$ and black corresponds to $S=0.5 \times 10^{-4}$.

in Figure 6. It can be clearly observed on the left figure that all the techniques yield the same order of convergence. In the right figure, where the techniques (ii), (iii) and (iv) are normalized with respect to (i), a clear and almost constant ratio can be observed between the different strategies. Whatever the number of degrees of freedom, the proposed technique therefore seems to reduce the error by $25 \%$ with respect to the homogeneous mesh, which is an additional $10 \%$ with respect to what would be obtained with a refinement based on constant parameter field.
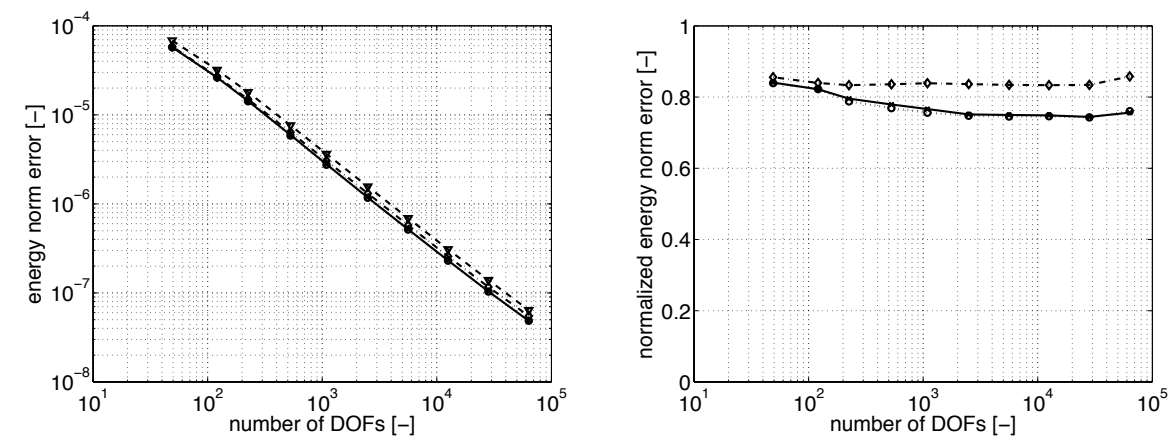

Fig. 6 (Left): Evolution of the error in energy norm with respect to the number of DOFs using: (i) the uniform mesh (dashed lines and triangles), and the adaptive meshes obtained using (ii) the solution $u_{\text {ref }_{0}}^{\prime \prime}$ for the average property field $a_{0}$ (dashed-dotted lines and diamonds), (iii) the reference solution $u_{\text {ref }}^{\prime \prime}$ (dotted lines and circles) and (iv) the proposed error indicator (solid lines and crosses). (Right): Plots (ii), (iii) and (iv) normalized by (i).

The conclusion of this example is that the proposed technique seems to perform exactly as well as an estimator based on the reference solution, and provide a large reduction of the error with the same number of DOFs with respect to a uniform mesh. 
4.3 Stochastic fluctuations of the properties

In this last example, we consider the same square domain $\Omega=[0,1] \times[0,1]$ as before, and the material property field is now a random field. Its first-order marginal distribution is log-normal, with average $a_{0}=2$ and variance $\sigma^{2}=1$ and its power-spectrum distribution is triangular with a correlation length $\ell_{c}=1 / 3$. Two realizations of the random field are plotted in Figure 7). As before, homogeneous Dirichlet boundary conditions are considered on the left and right sides $(x=0$ and $x=1$ ) and homogeneous Neumann boundary conditions are considered elsewhere (all conditions enforced almost surely). The same (deterministic) volume load is considered $f(x)=x^{2}-1 / 3$. We compute a reference solution on a refined mesh with 360,000 elements and using $N_{\mathrm{mc}}=10,000$ Monte Carlo trials, and denote it $u_{\text {ref }}(x)$. We also compute a reference solution considering the exact same problem, but a homogeneous field of material parameter at the value $a_{0}$. This solution is denoted $u_{\mathrm{ref}_{0}}$. Note that $u_{\mathrm{ref}}(x)$ is stochastic while $u_{\mathrm{ref}_{0}}$ is deterministic.
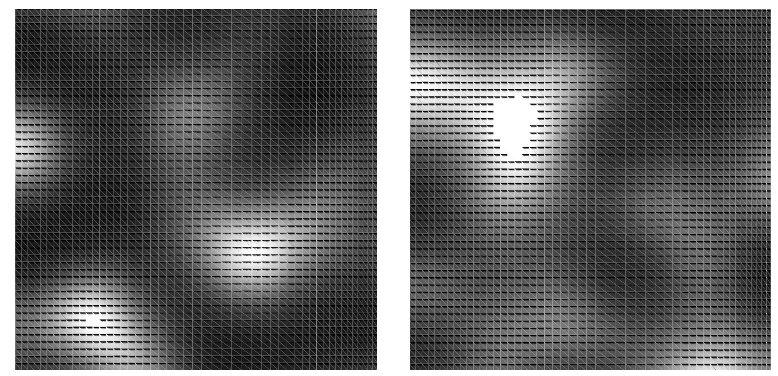

Fig. 7 Maps of two different realizations of the parameter $a(x)$. White corresponds to $a(x)=6$ and black corresponds to $a(x)=0$ (note that $a(x)>0$ everywhere).

Before looking at stochastic approximations of $u_{\mathrm{ref}}(x)$ and related quantities of interest (QoIs), we first compute the solutions for two different realizations of the property field using the same strategies as before: (i) a uniform mesh, (ii) a mesh deformed with respect to the curvature $u_{\text {ref }_{0}}^{\prime \prime}$, (iii) a mesh deformed with respect to the curvature $u_{\text {ref }}^{\prime \prime}$, and (iv) a mesh deformed with the proposed adaptivity process. Considering 2,500 elements and $h_{\max }=1.5$, we plot in Figure 8 the meshes obtained with the last three strategies (the upper row corresponds to the realization at the left of Figure 7 and the lower to the other one). As expected, there is no difference between the two meshes obtained for strategy (ii). Indeed, $u_{\text {ref }_{0}}^{\prime \prime}$ is the same for the two realizations and only contains information on the loading and boundary conditions. Interestingly, the meshes obtained with strategies (iii) and (iv) are very similar for each realization and vary widely between two realizations. Note again, as in the previous application, that there does not seem to be any way of guessing intuitively these apparently optimal meshes.

We now turn to stochastic evaluation of the solutions obtained with the different strategies. We start by observing the distributions of errors in the energy norm as well as in a particular QoI, which is the flux averaged over part of the 

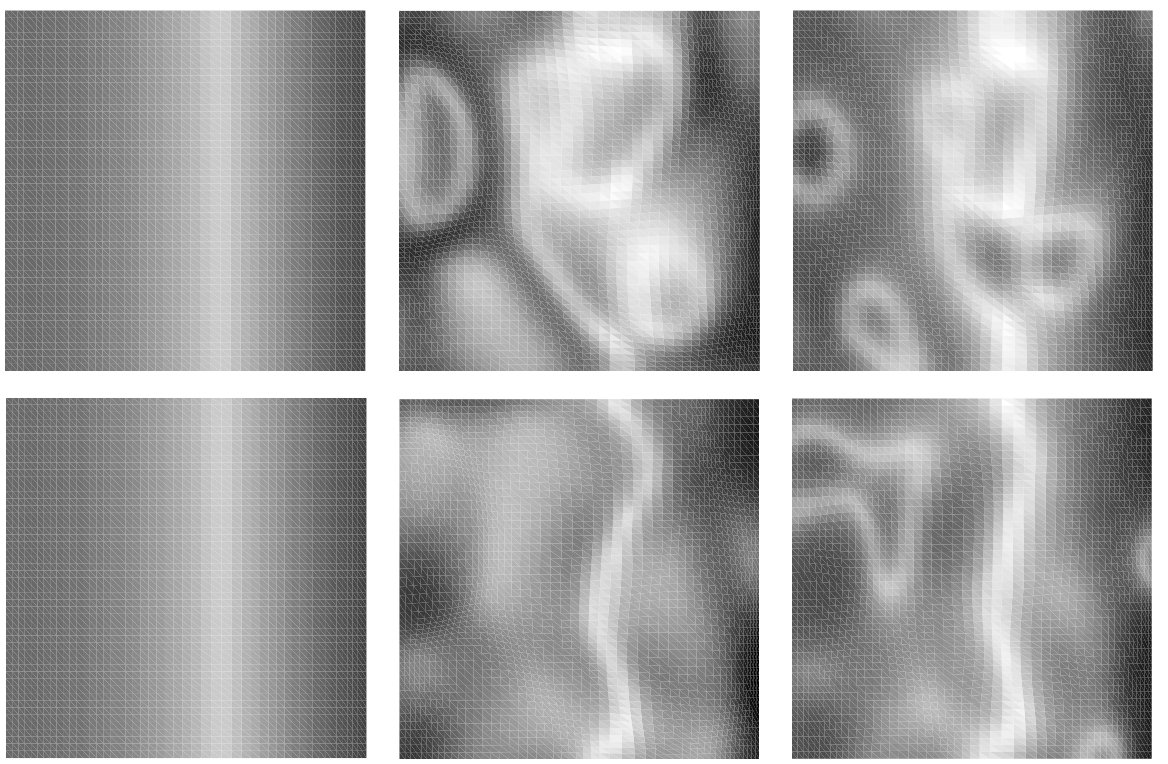

Fig. 8 Map of element areas obtained with 2,500 elements, $h_{\max }=1.5$, two different realizations of the parameter field $a(x)$ (the upper row corresponds to the left of Figure 7 and the lower row corresponds to the right of Figure 7) and the three adaptive strategies, using: (left) the solution $u_{\text {ref }_{0}}^{\prime \prime}$ for the average property field $a_{0}$, (center) the reference solution $u_{\mathrm{ref}}^{\prime \prime}$ and (right) the proposed error indicator. White corresponds to $S=3.5 \times 10^{-4}$ and black corresponds to $S=0.5 \times 10^{-4}$.

boundary:

$$
Q=\int_{y=0.8}^{1}(a(x) \nabla u \cdot n)_{\mid x=1} d y,
$$

where $n$ is the outgoing normal to the boundary. The distribution of errors in the energy norm are plotted at the left of Figure 9, while the distributions of errors in the QoI are plotted at its right. We also report in Table 1 the average and variance of the different errors. Both in the figure and in the table, the errors are normalized with respect to the average obtained with the homogeneous mesh. The objective is to insist on the potential improvements obtained using the strategy proposed in this paper with respect to the classical strategy which consists in using a uniform mesh. For both errors, it is noticeable that the proposed strategy indeed improves the estimation of the global solution, as well as that of the QoI. It also improves on the other possibility that would consist in using the mesh modified with respect to the average property field. Finally the proposed strategy does almost as well as the strategy using the (non-computable) $u_{\text {ref }}^{\prime \prime}$.

It is particularly interesting to note that the errors in the QoI possess bias, that is to say the average of the error does not vanish. As the error in the QoI is signed, one might have intuitively expected that its average would vanish. The results presented above show that, using a given mesh and a large number of Monte Carlo trials, the approximation of the average of the solution will converge to an erroneous value. We further study this effect by considering the convergence of the error made on the estimations of the mean and variance of the QoI using the 
Table 1 Average and variance of the normalized energy norm error and normalized QoI error using: (i) the uniform mesh, (ii) the solution $u_{\text {ref }_{0}}^{\prime \prime}$ for the average property field $a_{0}$, (iii) the reference solution $u_{\text {ref }}^{\prime \prime}$ and (iv) the proposed error indicator. The errors are normalized with respect to the average obtained with (i) and $10^{4}$ trials.

\begin{tabular}{|c|c|c|c|c|}
\hline Mesh & uniform & using $u_{\text {ref }_{0}}^{\prime \prime}$ & using $u_{\text {ref }}^{\prime \prime}$ & proposed \\
\hline Average of error in EN & 1.00 & 0.78 & 0.64 & 0.68 \\
Variance of error in EN & 0.20 & 0.09 & 0.05 & 0.06 \\
\hline Average of error in QoI & 1.00 & 0.61 & 0.47 & 0.51 \\
Variance of error in QoI & 0.75 & 0.30 & 0.11 & 0.17 \\
\hline
\end{tabular}
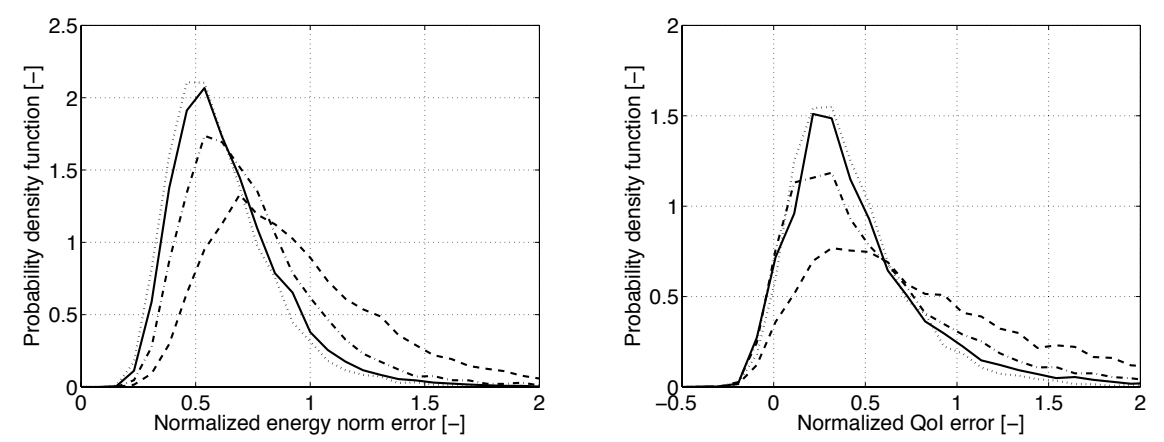

Fig. 9 Histograms of normalized energy norm error (left) and normalized QoI error (right) using: (i) the uniform mesh (dashed lines), (ii) the solution $u_{\mathrm{ref}_{0}}^{\prime \prime}$ for the average property field $a_{0}$ (dashed-dotted lines), (iii) the reference solution $u_{\text {ref }}^{\prime \prime}$ (dotted lines) and (iv) the proposed error indicator (solid lines). The errors are normalized with respect to the average of (i) for $10^{4}$ trials.

different strategies and increasing numbers of Monte Carlo trials. The convergence study for the QoI mean is performed in the following way: (1) for a given number of Monte Carlo trials $N_{\mathrm{mc}}$, we pick a large set of $N_{\mathrm{mc}}$ independent realizations of the properties; (2) for each element in the set, we estimate the mean of the QoI, and (3) by averaging over the different elements in the set, we estimate a mean and variance for the mean QoI with $N_{\mathrm{mc}}$ Monte Carlo trials. This sequence is repeated for different values of $N_{\mathrm{mc}}$ and the results are plotted at the left of Figure 10. Finally the same study is performed for the variance of the QoI and plotted at the right of Figure 10. It is clear on this figure that, although it does not vanish, the biases in the estimators of the mean and variance of the QoI are largely reduced when using the strategy described in this paper.

\section{Conclusion}

The proposed $r$-adaptivity strategy aims at finding the best fitted FE mesh for different realizations of the perturbed material properties field. It is based on an a-priori computable error indicator accounting for the effect of the perturbation of the material properties in the second derivatives of the solution. Then, this error indicator is introduced in an $a d-h o c$ remeshing criterion to derive the stretching 

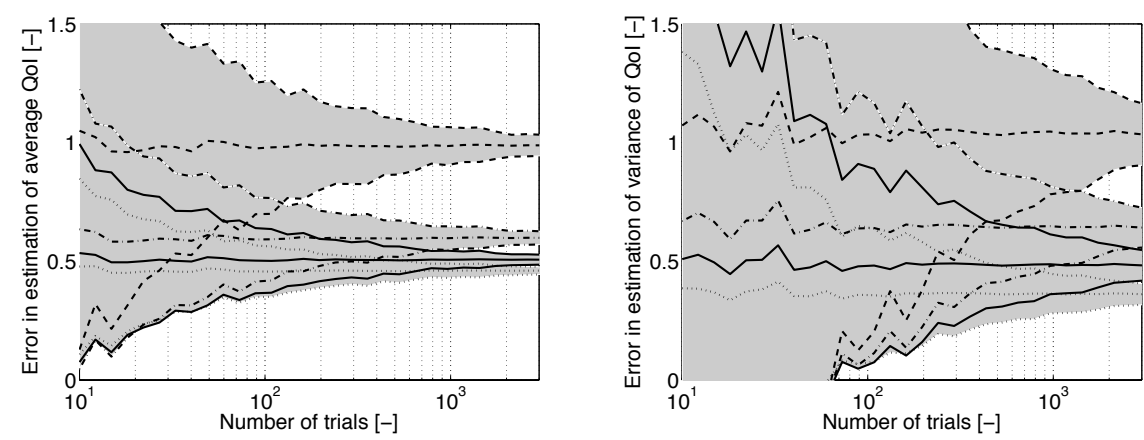

Fig. 10 Convergence of the error in the estimators of the mean (left) and variance (right) for the QoI using: (i) the uniform mesh (dashed lines), (ii) the solution $u_{\text {ref }_{0}}^{\prime \prime}$ for the average property field $a_{0}$ (dashed-dotted lines), (iii) the reference solution $u_{\text {ref }}^{\prime \prime}$ (dotted lines) and (iv) the proposed error indicator (solid lines). The grey shades indicate $90 \%$ confidence intervals. The errors are normalized with respect to (i) for $10^{4}$ trials.

factor to be enforced to each element of the mesh in order to optimally relocate the nodes. This expansion or contraction factor produces the movement of the nodes. The methodology adopted here to move the mesh is based on solving a linear elastic model with enforced elementary pre-strains.

When used in the context of a Monte Carlo or collocation sampling process for solving a stochastic problem, this strategy has a marginal computational cost at each sample and improves the accuracy of the resulting statistics. On all tested cases, the biases of the statistical estimations were reduced, as a consequence of the small and consistent improvements obtained for each sample.

Acknowledgements Part of this research was conducted while the second author was staying at École Centrale Paris as an invited Professor. Both the authors would like to thank École Centrale Paris for this invitation.

\section{References}

1. Ainsworth, M., Oden, J.T.: A posteriori error estimation in finite element analysis. Pure and Applied Mathematics. Wiley-Interscience (2000)

2. Alves Fernandes, V., Lopez-Caballero, F., Costa d'Aguiar, S.: Probabilistic analysis of numerical simulated railway track global stiffness. Comp. \& Geotech. 55, 267-276 (2014). DOI 10.1016/j.compgeo.2013.09.017

3. Askes, H., Rodriguez-Ferran, A.: A combined rh-adaptive scheme based on domain subdivision. Formulation and linear examples. Int. J. Numer. Meth. Engr. 51(3), 253-273 (2001). DOI 10.1002/nme.142

4. Babuška, I., Nobile, F., Tempone, R.: A stochastic collocation method for elliptic partial differential equations with random input data. SIAM Rev. 52(2), 317-355 (2007). DOI $10.1137 / 100786356$

5. Babuška, I., Rheinboldt, W.C.: Error estimates for adaptive finite element computations. SIAM J. Numer. Anal. 15(4), 736-755 (1978)

6. Babuška, I., Rheinboldt, W.C.: A posteriori error estimates for the finite element method. Int. J. Numer. Meth. Engr. 12(10), 1597-1615 (1978) 
7. Babuška, I., Tempone, R., Zouraris, G.E.: Galerkin finite element approximations of stochastic elliptic partial differential equations. SIAM J. Numer. Anal. 42(2), 800-825 (2004). DOI 10.1137/S0036142902418680

8. Babuška, I., Whiteman, J., Strouboulis, T.: Finite elements: an introduction to the method and error estimation. Oxford University Press (2010)

9. Baydoun, I., Savin, E., Cottereau, R., Clouteau, D., Guilleminot, J.: Kinetic modeling of multiple scattering of elastic waves in heterogeneous anisotropic media. Wave Motion (2014). DOI 10.1016/j.wavemoti.2014.08.001. Accepted for publication

10. Bespalov, A., Powell, C.E., Silvester, D.: A priori error analysis of stochastic Galerkin mixed approximations of elliptic PDEs with random data. SIAM J. Numer. Anal. 50(4), 2039-2063 (2012). DOI 10.1137/110854898

11. Butler, T., Dawson, C., Wildey, T.: A posteriori error analysis of stochastic differential equations using polynomial chaos expansions. SIAM J. Sci. Comp. 33(3), 1267-1291 (2011). DOI 10.1137/100795760

12. Caflisch, R.E.: Monte Carlo and quasi-Monte Carlo methods. Acta Numerica 7, 1-49 (1998). DOI 10.1017/S0962492900002804

13. Chamoin, L., Florentin, E., Pavot, S., Visseq, V.: Robust goal-oriented error estimation based on the constitutive relation error for stochastic problems. Comp. \& Struct. 106-107, 189-195 (2012). DOI 10.1016/j.compstruc.2012.05.002

14. Charrier, J.: Strong and weak error estimates for elliptic partial differential equations with random coefficients. SIAM J. Numer. Anal. 50(1), 216-246 (2012). DOI $10.1137 / 100800531$

15. Charrier, J., Scheichl, R., Teckentrup, A.L.: Finite element error analysis of elliptic PDEs with random coefficients and its application to multilevel Monte Carlo methods. SIAM J. Numer. Anal. 51(1), 322-352 (2013). DOI 10.1137/110853054

16. Cho, M., Jun, S.: r-adaptive mesh generation for shell finite element analysis. J. Comp. Phys. 199(1), 291-316 (2004). DOI 10.1016/j.jcp.2004.02.007

17. Choi, S.K., Gandhi, R.V., Canfield, R.A.: Reliability-based structural design. Springer (2006)

18. Chorin, A.: Gaussian fields and random flow. J. Fluid Mech. 63, 21-32 (1974)

19. Clouteau, D., Cottereau, R., Lombaert, G.: Dynamics of structures coupled with elastic media - a review. J. Sound Vib. 332(10), 2415-2436 (2013). DOI 10.1016/j.jsv.2012.10.011

20. Cottereau, R.: Numerical strategy for unbiased homogenization of random materials. Int. J. Numer. Meth. Engr. 95(1), 71-90 (2013). DOI http://dx.doi.org/10.1002/nme.4502

21. Cottereau, R., Clouteau, D., Soize, C.: Probabilistic impedance of foundation: impact of the seismic design on uncertain soils. Earth. Engr. Struct. Dyn. 196(17-20), 899-918 (2007). DOI 10.1002/eqe.794

22. Deb, M.K., Babuška, I., Oden, J.T.: Solution of stochastic partial differential equations using Galerkin finite element techniques. Comp. Meth. Appl. Mech. Engr. 190(48), 63596372 (2001). DOI 10.1016/S0045-7825(01)00237-7

23. Debusschere, B., Najm, H.N., Matta, A., Knio, O., Ghanem, R., le Maître, O.: Protein labeling reactions in electrochemical microchannel flow: numerical prediction and uncertainty propagation. Phys. Fluids 15(8), 2238-2250 (2003)

24. Efendiev, Y., Pankov, A.: Numerical homogenization of nonlinear random parabolic operators. SIAM Multiscale Model. Simul. 2(2), 237-268 (2004). DOI 10.1137/030600266

25. Florentin, E., Díez, P.: Adaptive reduced basis strategy based on goal oriented error assessment for stochastic problems. Comp. Meth. Appl. Mech. Engr. 225-228, 116-127 (2012). DOI 10.1016/j.cma.2012.03.016

26. Foo, J., Wan, X., Karniadakis, G.E.: The multi-element probabilistic collocation method (ME-PCM): Error analysis and applications. J. Comp. Phys. 227(22), 9572-9595 (2008). DOI 10.1016/j.jcp.2008.07.009

27. Frankel, A., Clayton, R.W.: A finite-difference simulation of wave propagation in twodimensional random media. Bull. Seismological. Soc. Amer. 74(6), 2167-2186 (1984)

28. Ghanem, R.G., Spanos, P.D.: Stochastic finite elements: a spectral approach. SpringerVerlag (1991)

29. Grajewski, M., Köster, M., Turek, S.: Numerical analysis and implementational aspects of a new multilevel grid deformation method. Appl. Numer. Math. 60(8), 767-781 (2010). DOI 10.1016/j.apnum.2010.03.017

30. Holliger, K.: Upper-crustal seismic velocity heterogeneity as derived from a variety of P-wave sonic logs. Geophys. J. Int. 125(3), 813-829 (1996). DOI 10.1111/j.1365- 
246X.1996.tb06025.x

31. Huerta, A., Rodriguez-Ferran, A., Díez, P., Sarrate, J.: Adaptive finite element strategies based on error assessment. Int. J. Numer. Meth. Engr. 46(10), 1803-1818 (1999). DOI 10.1002/(SICI)1097-0207(19991210)46:10¡1803::AID-NME725¡3.0.CO;2-3

32. Huyse, L., Maes, M.A.: Random field modeling of elastic properties using homogenization. J. Engr. Mech., ASCE 127(1), 27-36 (2001). DOI 10.1061/(ASCE)07339399(2001)127:1(27)

33. Jehel, P., Cottereau, R.: On damping created by heterogeneous yielding in the numerical analysis of nonlinear RC frame elements. Comp. \& Struct. (2014). Submitted for publication

34. Ladevèze, P., Florentin, E.: Verification of stochastic models in uncertain environments using the constitutive relation error method. Comp. Meth. Appl. Mech. Engr. 196(1-3), 225-234 (2006). DOI 10.1016/j.cma.2006.03.006

35. Ladevèze, P., Pelle, J.P.: Mastering calculations in linear and nonlinear mechanics. Mechanical Engineering. Springer (2005)

36. Le Bris, C.: Some numerical approaches for weakly random homogenization. In: G.K. et al. (ed.) Numerical Mathematics and Advanced Applications 2009, pp. 29-45. Springer-Verlag (2010). DOI 10.1007/978-3-642-11795-4_3

37. Le Maître, O.P., Knio, O.M.: Spectral methods for uncertainty quantification: with applications to computational fluid dynamics. Springer (2010)

38. Littlefield, D.L.: The use of r-adaptivity with local, intermittent remesh for modeling hypervelocity impact and penetration. Int. J. Impact Engr. 26(1-10), 433-442 (2001). DOI 10.1016/S0734-743X(01)00093-8

39. Materna, D., Barthold, F.J.: Goal-oriented r-adaptivity based on variational arguments in the physical and material spaces. Comp. Meth. Appl. Mech. Engr. 198(41-44), 3335-3351 (2009). DOI 10.1016/j.cma.2009.06.015

40. Mathelin, L., le Maître, O.: Dual-based a posteriori error estimate for stochastic finite element methods. Comm. Appl. Math. Comp. Sci. 2(1), 83-115 (2007). DOI 10.2140/camcos.2007.2.83

41. Matthies, H.G.: Stochastic finite elements: computational approaches to stochastic partial differential equations. J. Appl. Math. Mech. 88(11), 849-873 (2008). DOI 10.1002/zamm.200800095

42. Oden, J.T., Babuška, I., Nobile, F., Feng, Y., Tempone, R.: Theory and methodology for estimation and control of errors due to modeling, approximation, and uncertainty. Comp. Meth. Appl. Mech. Engr. 194(2-5), 195-204 (2005). DOI 10.1016/j.cma.2003.06.003

43. Ostoja-Starzewski, M.: Microstructural randomness and scaling in mechanics of materials. CRC Press (2007)

44. Peraire, J., Vahdati, M., Morgan, K., Zienkiewicz, O.C.: Adaptive remeshing for compressible flow computations. J. Comp. Phys. 72(2), 449-466 (1987). DOI 10.1016/00219991(87)90093-3

45. Phoon, K.K.: Reliability-based design in geotechnical engineering. CRC Press (2008)

46. Piggott, M.D., Pain, C.C., Gorman, G.J., Power, P.W., Goddard, A.J.: h, r, and hr adaptivity with applications in numerical ocean modelling. Ocean Modelling 10(1-2), 95-113 (2005). DOI 10.1016/j.ocemod.2004.07.007

47. Popescu, R., Deodatis, G., Nobahar, A.: Effects of random heterogeneity of soil properties on bearing capacity. Prob. Engr. Mech. 20, 324-341 (2005). DOI 10.1016/j.probengmech.2005.06.003

48. Rajagopal, A., Sivakumar, S.M.: A combined r-h adaptive strategy based on material forces and error assessment for plane problems and bimaterial interfaces. Comp. Mech. 41(1), 49-72 (2007). DOI 10.1007/s00466-007-0168-8

49. Robert, C.P., Casella, G.: Monte Carlo statistical methods. Springer (2004)

50. Sato, H., Fehler, M.C., Maeda, T.: Seismic wave propagation and scattering in the heterogeneous earth, second edn. Springer (2012)

51. Shi, W., Zhang, C.: Error analysis of generalized polynomial chaos for nonlinear random ordinary differential equations. Appl. Numer. Math. 62(12), 1954-1964 (2012). DOI 10.1016/j.apnum.2012.08.007

52. Stefanou, G.: The stochastic finite element method: past, present and future. Comp. Meth. Appl. Mech. Engr. 198(9-12), 1031-1051 (2009). DOI 10.1016/j.cma.2008.11.007

53. Ta, Q.A., Clouteau, D., Cottereau, R.: Modeling of random anisotropic elastic media and impact on wave propagation. Europ. J. Comp. Mech. 19(1-3), 241-253 (2010). DOI 
10.3166/ejcm.19.241-253

54. Taherzadeh, R., Clouteau, D., Cottereau, R.: Simple formulas for the dynamic stiffness of pile groups. Earth. Engr. Struct. Dyn. 38(15), 1665-1685 (2009). DOI 10.1002/eqe.918

55. Wiberg, N.E., Díez, P.: Adaptive modeling and simulation. Comp. Meth. Appl. Mech. Engr. 195(4-6), 205-480 (2006)

56. Yang, Z.J., Su, X.T., Chen, J.F., Liu, G.H.: Monte Carlo simulation of complex cohesive fracture in random heterogeneous quasi-brittle materials. Int. J. Solids Struct. 46, 32223234 (2009). DOI 10.1016/j.ijsolstr.2009.04.013

57. Zhang, G., Gunzburger, M.: Error analysis of a stochastic collocation method for parabolic partial differential equations with random input data. SIAM J. Numer. Anal. 50(4), 19221940 (2012). DOI 10.1137/11084306X

58. Zienkiewicz, O.C., Zhu, J.Z.: A simple error estimator and adaptive procedure for practical engineering analysis. Int. J. Numer. Meth. Engr. 24, 337-357 (1987) 